\title{
Effect of processing of bovine milk on gastrointestinal symptoms and intestinal pressure in sensitive individuals
}

\author{
Anu Nuora ${ }^{1}$, Tuomo Tupasela ${ }^{2}$, Johanna Jokioja ${ }^{1}$, Raija Tahvonen ${ }^{2}$, Heikki Kallio ${ }_{4}$, \\ Baoru Yang ${ }^{1}$, Susanna Rokka ${ }^{2}$, Pertti Marnila ${ }^{2}$, Petri Mäkelä ${ }^{3}$, Jonne Pohjankukka ${ }^{4}$, \\ Tapio Pahikkala ${ }^{4}$, Matti Viitanen ${ }^{5,6}$ and Kaisa Linderborg ${ }^{1}$ \\ ${ }^{1}$ Food Chemistry and Food Development, University of Turku, Turku, Finland, \\ ${ }^{2}$ Bio-Based Business and Industry, Natural Resources Institute Finland, Jokioinen, Finland, \\ ${ }^{3}$ Endoscopy Unit, Turku City Hospital, Turku, Finland, \\ ${ }^{4}$ Department of Information Technology, University of Turku, Turku, Finland, \\ ${ }^{5}$ Department of Geriatrics, University of Turku, Turku City Hospital, Turku, and \\ ${ }^{6}$ Department of Geriatrics, Karolinska Institutet, Karolinska University Hospital Huddinge, Stockholm, Sweden
}

\begin{abstract}
Over the last years, the consumption of unpasteurised milk has increased in popularity in the Western countries, despite the known risks associated with food-borne pathogens. Some people appear to experience milk-related gastrointestinal symptoms even when tested negative for lactose intolerance and milk allergy. In such cases, processing of milk, most often homogenisation or heat treatment, has been suggested to be involved in the induction of the gastrointestinal symptoms. Commercial milk is generally homogenised for the purpose of physical stability. Homogenisation reduces the size of fat droplets. Breaking of the fat globules creates new interface, which is covered with denaturated proteins. Further denaturation level of the proteins depends on the severity of the heat treatment. In this study entity, the effect of processing of bovine milk on the gastrointestinal symptoms in self-reported milk sensitive individuals was tested in two separate double blind crossover trials. Tested milks included raw milk (unprocessed), pasteurized milk, pasteurized and homogenized milk, and UHT treated and homogenized milk thus including milks at both end of commercially approved liquid milks in Finland. All milks came from the same herd of cows, which nulled the effect of genetic background and feeding of the cows. Ingestible $\mathrm{pH}$-, pressure and temperature measuring probe was used to investigate gastrointestinal pressure. Self-reported diaries were used to measure perceived gastrointestinal symptoms. Additionally inflammation markers were studied from the plasma. In the first trial no significant difference was found in the amount of gastrointestinal symptoms or in the intestinal pressure after the consumption of native and processed milk. However, the obtained results on pressure in the large intestine $(\mathrm{P}=0.068)$ as well as reported symptoms $(\mathrm{P}=0.103)$ suggested that further studies in this area were needed. However, in the second trial where pasteurised, pasteurised and homogenised, and UHT -treated and homogenized milks were compared, the amount, severity or duration of the reported symptoms did not differ. This study demonstrates that the ingestible pressure-, $\mathrm{pH}$ and temperature measuring probe provided a novel possibility to study intestinal pressure in nutrition trials. The results of the present studies do not support the hypothesis that cows' milk processing would induce gastrointestinal symptoms in milk sensitive but lactose tolerant subjects.
\end{abstract}

\section{Conflict of Interest}

There is no conflict of interest 